Supplementary information 2 for

\section{A Dual Sensor Spin Trap for use with EPR Spectroscopy}

\section{Stuart T. Caldwell, Caroline Quin, Ruth Edge}

\section{and Richard C. Hartley}

WestCHEM Department of Chemistry, University of Glasgow,

Glasgow, G12 8QQ, UK and EPSRC National EPR Service, School

of Chemistry, University of Manchester, Oxford Road, Manchester,

M13 9PL, UK.

\section{Comparison of Spectrum and Simulation of Nitroxyl Radical 5, Generated from Nitrone 1 and Iron(III) Chloride}

\section{Comments}

The experimental spectrum of nitroxyl radical 5 was simulated using the $A_{\mathrm{N}}$ and $A_{\mathrm{H}}{ }^{\beta}$ hyperfine couplings found for the tricyclic deuterated nitroxyl radical 15 and adding two additional hyperfine couplings of about $1 \mathrm{G}$. The best fit obtained had these additional couplings set to 0.99 and $1.05 \mathrm{G}$ and is shown superimposed on the experimentally obtained spectrum on the following page. The resonance fields of the peaks superimpose on those in the experimental spectrum. These small hyperfine couplings can be varied by about $0.05 \mathrm{G}$ with reasonable fit to the experimental spectrum, hence although setting them subtly different values gives a slightly better lineshape, they may be the same within experimental error. Although, we have included crude variable linewidth effects in the spectrum by standard methods, we have not attempted to rigorously model these tumbling effects.

Simulation values

Hyperfine $\mathrm{N}=15.70 \mathrm{G}, \mathrm{H}=1.42,1.05,0.99 \mathrm{G}$

Lineshape $=0.7$ Lorentzian +0.3 Gaussian.

Linewidth with tumbling: $\mathrm{a}=0.47, \mathrm{~b}=-0.3, \mathrm{c}=0.3$.

$\mathrm{g}$ factor $=2.0055$ corrected to strong pitch, Freq/MA etc as experimental conditions. 


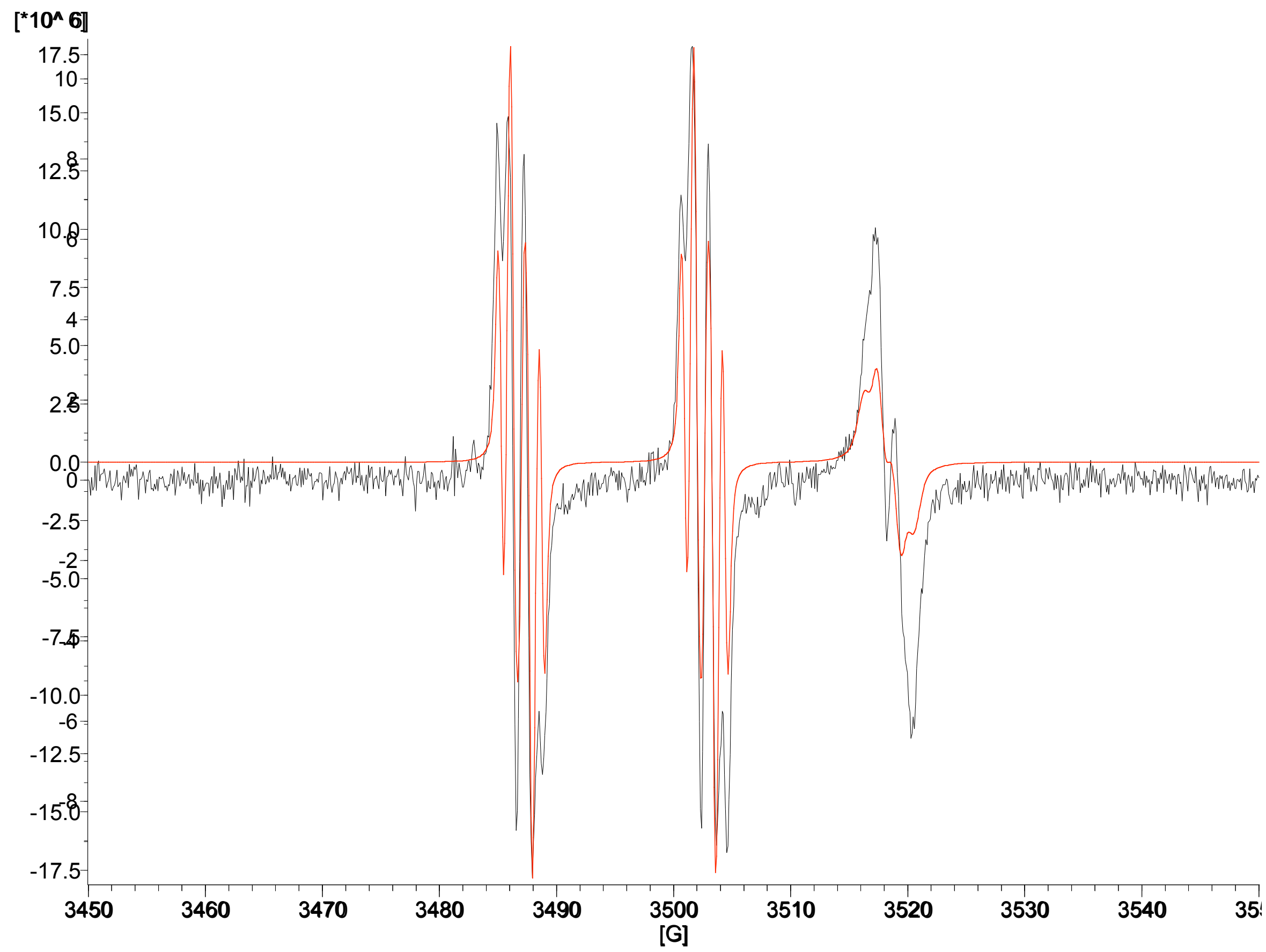

